\title{
Spinal Stenosis Presenting with Scrotal and Perianal Claudication
}

\author{
Jacob YL Oh, Jun-Hao Tan, Timothy WW Teo, Hwan-Tak Hee \\ Department of Orthopaedic Surgery, Khoo Teck Puat Hospital, Singapore
}

\begin{abstract}
A 63-year-old gentleman presented with a one-year duration of progressive neurogenic claudication. However, unlike most patients who presents with leg symptoms, his pain was felt in his scrotal and perianal region. This was exacerbated with walking and standing, but he had immediate relief with sitting. An magnetic resonance imaging (MRI) was performed which showed severe central canal stenosis. An $L 3 / 4$ and $L 4 / 5$ surgical decompression and a transforaminal lumbar interbody fusion was performed, and the patient made good recovery with immediate resolution of symptoms. Although rare, spinal stenosis should be considered a differential when approaching a patient with perianal and scrotal claudication, even in the absence of leg claudication. An MRI is useful to confirm the diagnosis. This rare symptom may be a sign of severe cauda equina compression and we recommend decompression with predictable good results.
\end{abstract}

Keywords: Spinal; Stenosis; Atypical; Claudication

\section{Introduction}

Spinal stenosis classically presents with neurogenic claudication. Patients typically complain of leg pain that is exacerbated with extension of the spine, while flexion relieves the symptoms. Atypical presentations of spinal stenosis have been described, including intermittent priaprism [1,2] and a bilateral foot drop [3]. The purpose of this study is to highlight an atypical presentation of spinal stenosis presenting as scrotal and perianal claudication, and discuss the approach to management

\section{Case Report}

A 63-year-old Asian male with no significant past medical history presented to our clinic with a 12 -month history of progressive perianal and scrotal claudication. $\mathrm{He}$ had minimal back pain. The patient was very disabled by the claudication in his perianal and scrotal region. He was unable to stand or walk for more than 5 minutes, and had to sit or lean forward to relieve his symptoms. He denied symptoms of leg claudication or bowel and bladder dysfunction. Neurological examination of the spine revealed no back tenderness and a good range of motion. Power at all myotomes was full (Medical Research Council grade 5). Sensory examination was normal. Reflexes were normal with down going plantars. The numbness was localized to the perineum/scrotal region. Peripheral pulses were present and a digital rectal examination performed was unremarkable.

Radiographs showed lumbar spondylosis with a grade 1 spondylolisthesis at L3/L4, and L4/L5 (Figs. 1, 2). magnetic resonance imaging (MRI) revealed severe disc degeneration at L3/4 and L4/5 with severe central canal

Received Feb 12, 2014; Revised Mar 4, 2014; Accepted Mar 4, 2014

Corresponding author: Jacob YL Oh

Department of Orthopaedic Surgery, Khoo Teck Puat Hospital, 90 Yishun Central, Singapore 768828

Tel: +6591555918, Fax: +6591555918, E-mail: Jacob_oh@yahoo.com 
stenosis, worse at L4/L5, with compression of the cauda equina (Figs. 3, 4). A referral to the Urologist was also made to exclude other urogenital causes but the tests were unremarkable. This included a physical examination, urodynamic testing and an ultrasound. In view of the patient's progressive symptoms and radiological findings, a decompression and fusion of the L3/4 and the L4/5 levels were performed for the patient's spinal stenosis. The surgery was uncomplicated.

Postoperatively, the patient recovered well with immediate resolution of his symptoms. At his 12-month follow up, his scrotal and perianal claudication had not recurred and he was able to exercise again, walking up to 1 hour a day.

\section{Discussion}

Spinal stenosis was first described by Verbiest [4] in 1954, classically presenting with discomfort in the thighs,

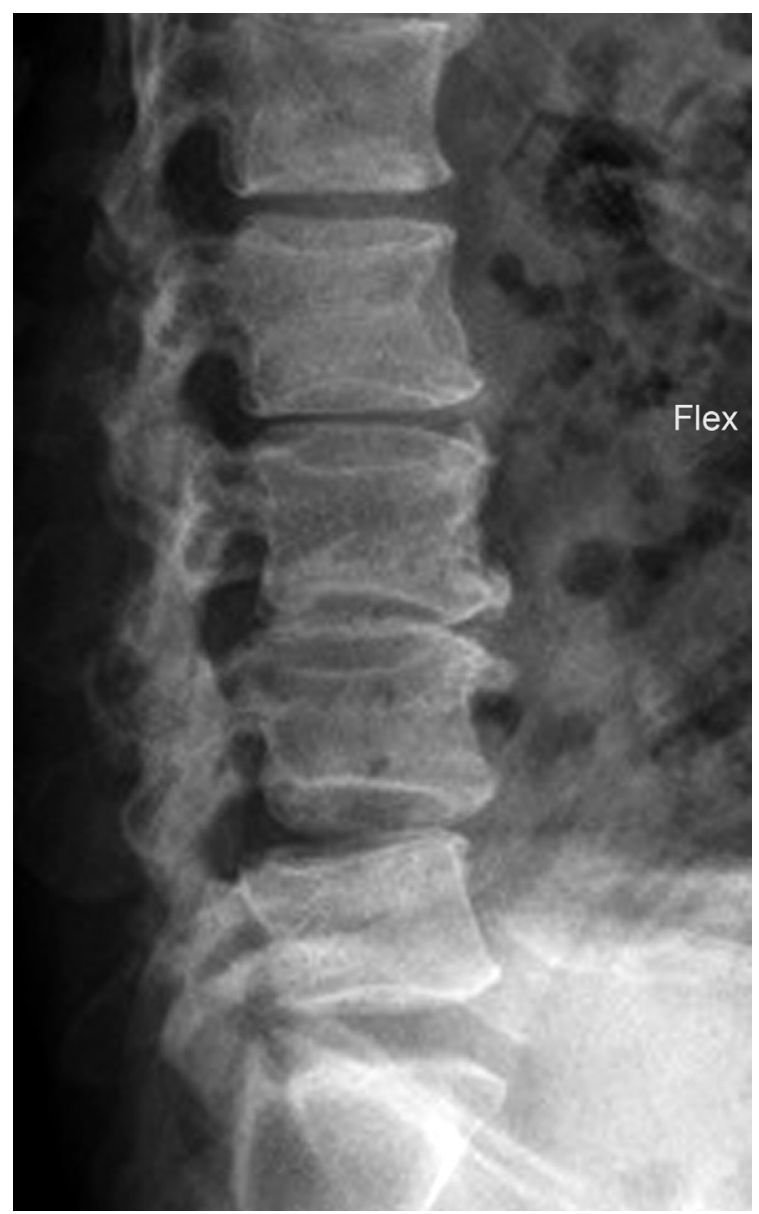

Fig. 1. Flexion view of the lumbar spine showing marked degenerative changes and pondylolisthesis at $\mathrm{L} 3 / 4$ and $\mathrm{L} 4 / 5$. calves and feet on walking. The pathophysiology of neurogenic claudication in lumbar spinal stenosis has been well described. This is believed to arise from ischemia of the lumbosacral nerve roots, as a result of increased metabolic demand during exercise. There is also co-existing vascular compromise of the nerve roots due to pressure from surrounding structures [5].

Our patient presented with atypical symptoms of spinal stenosis, with predominantly scrotal and perianal claudication, in the absence of leg claudication. Apart from the location, these symptoms were consistent with neurogenic claudication-relieved by squatting and sitting and aggravated with standing and walking. The MRI also confirmed spinal stenosis at the levels of L3/L4 and L4/ L5. Atypical presentations of spinal stenosis have been described, including intermittent priaprism $[1,2]$ and a bilateral foot drop [3]. To the best of our knowledge, spi-

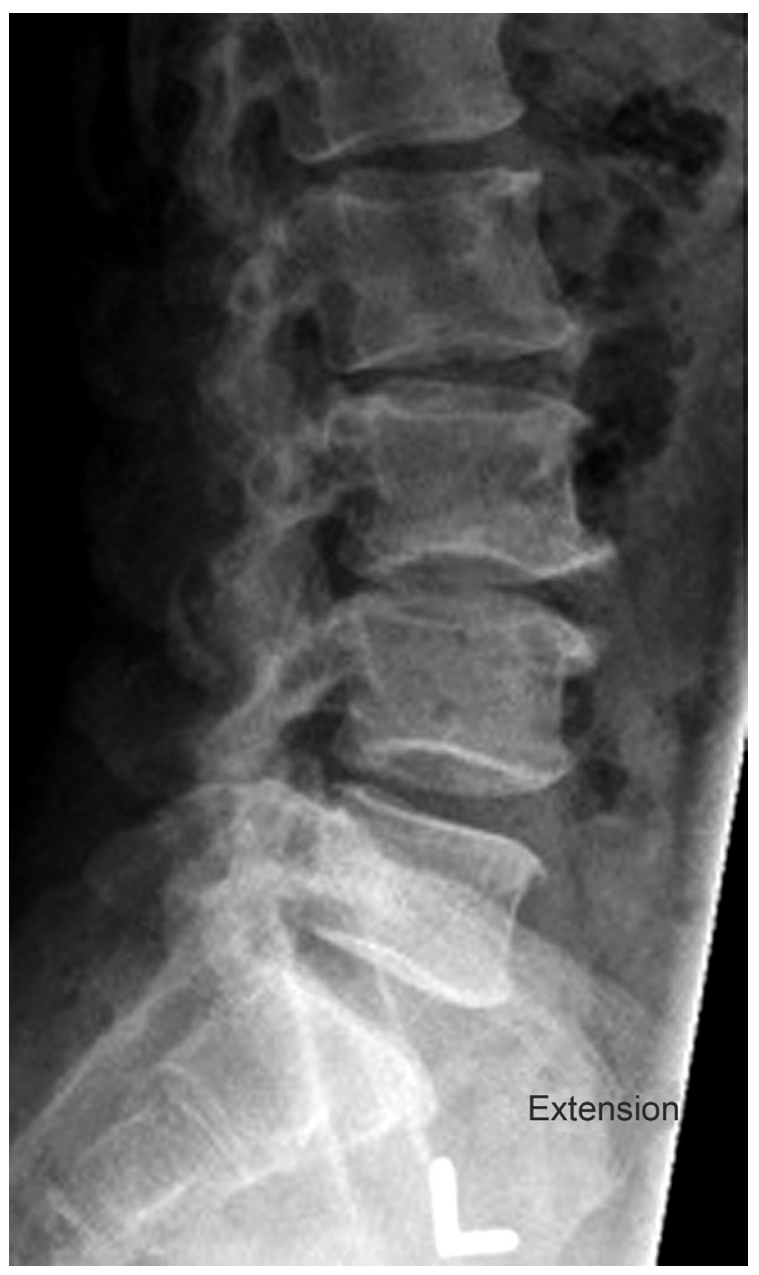

Fig. 2. Extension view of the lumbar spine showing reduction of the spondylolisthesis at $L 3 / 4$ and $L 4 / 5$ compared to the flexion view. 
nal stenosis causing scrotal and perianal claudication has never been reported.

Fraser et al. [6] reported that cauda equina syndrome

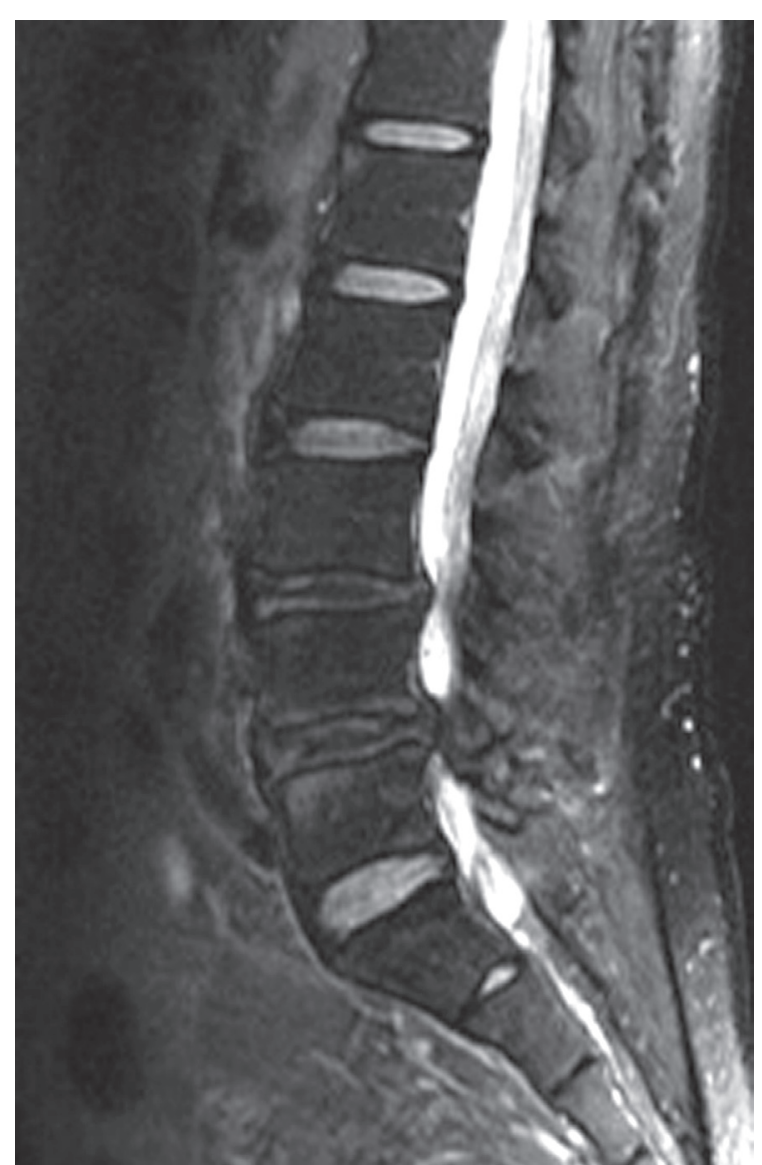

Fig. 3. Magnetic resonance imaging scan showing disc degeneration at $L 3 / 4$ and $L 4 / 5$ causing spinal stenosis.

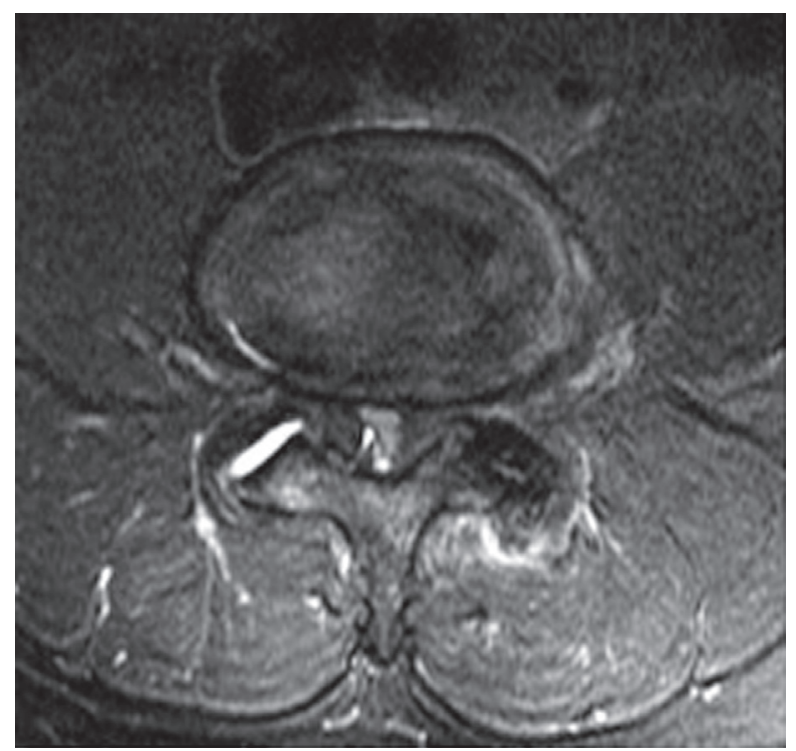

Fig. 4. Axial cut showing severe spinal stenosis at $L 4 / 5$. might present in a variety of ways. This includes bladder and bowel dysfunction, pain, sexual dysfunction, and neurological deficits in the lower limbs. Of these, saddle anaesthesia was found to be the most striking feature of cauda equina syndrome, which is the result of damage to the sensory nerves at the cauda equina. Due to the similarity of dermatomal distribution and complete resolution of symptoms after surgery, we believe that this atypical presentation of spinal stenosis may suggest severe cauda equina compression.

In conclusion, atypical presentation of spinal stenosis should be considered when a patient presents with perianal and scrotal claudication, even in the absence of leg claudication. An MRI is useful to confirm the diagnosis. This rare symptom may be a sign of severe cauda equina compression and we recommend decompression with predictable good results.

\section{Conflict of Interest}

No potential conflict of interest relevant to this article was reported.

\section{References}

1. Cansever T, Civelek E, Sencer A, Karasu A, Turantan I. Intermittent priapism in degenerative lumbar spinal stenosis: case report. Turk Neurosurg 2007;17:260-3.

2. Baba H, Maezawa Y, Furusawa N, Kawahara N, Tomita K. Lumbar spinal stenosis causing intermittent priapism. Paraplegia 1995;33:338-45.

3. Oluigbo CO, Qadri SR, Dardis R, Choksey MS. Lumbar canal stenosis presenting with acute bilateral foot drop. Br J Neurosurg 2006;20:87-9.

4. Verbiest H. A radicular syndrome from developmental narrowing of the lumbar vertebral canal. J Bone Joint Surg Br 1954;36:230-7.

5. Porter RW. Spinal stenosis and neurogenic claudication. Spine (Phila Pa 1976) 1996;21:2046-52.

6. Fraser S, Roberts L, Murphy E. Cauda equina syndrome: a literature review of its definition and clinical presentation. Arch Phys Med Rehabil 2009;90:19648. 